1 Universidade de Brasília (UnB) - Brasília (DF), Brasil.

fernandocupertino@gmail.com

2 Universidade de Brasília (UnB), Núcleo de Estudos em Saúde Pública (Nesp) Brasília (DF), Brasil.

jessicalopes.scunb@gmail.com

3 Universidade de Brasília (UnB), Núcleo de Estudos em Saúde Pública (Nesp) Brasília (DF), Brasil.

valeriamendonca@unb.br

4 Universidade de Brasília (UnB) - Brasília (DF),

Brasil.

fatimasousa@unb.br

\section{Acesso e equidade nos serviços de saúde: uma revisão estruturada}

\author{
Access and equity in health services: a structured review
}

Fernando Passos Cupertino de Barros ${ }^{\mathbf{1}}$, Jéssica de Souza Lopes² ${ }^{\mathbf{2}}$ Ana Valéria Machado

Mendonça ${ }^{3}$, Maria Fátima de Sousa ${ }^{4}$

RESUMO Artigo de revisão de literatura sobre a organização de serviços da Atenção Primária à Saúde em suas dimensões de acesso e equidade. Os artigos foram identificados nas bases de dados da Biblioteca Virtual em Saúde (Centro Latino-Americano e do Caribe de Informação em Ciências da Saúde - Bireme). Palavras-chave: 'acesso aos serviços de saúde' e 'equidade'; período: 1993 a 2013. Dos 31 artigos encontrados, 29 estão em português, um em inglês e um em espanhol, publicados por 16 diferentes periódicos; 18 artigos (58,06\%) referem-se à organização de serviços de saúde e 13 (41,94\%) à equidade. A maioria (80,64\%) utilizou abordagem qualitativa. Mais estudos são necessários a fim de avaliar se os sistemas de saúde tiveram impactos mensuráveis em termos de acesso e equidade.

PALAVRAS-CHAVE Acesso aos serviços de saúde. Equidade. Atenção Primária à Saúde.

ABSTRACT Literature review article on the organization of Primary Health Care services in its dimensions of access and equity. The articles were identified in the databases of the Virtual Health Library (Centro Latino-Americano e do Caribe de Informação em Ciências da SaúdeBireme); Keywords: 'access to health services' and 'equity'; period: 1993 to 2013. Of the 31 articles found, 29 are in portuguese, one in english and one in spanish, published by 16 different journals; 18 articles (58.06\%) refer to the organization of health services and 13 (41.94\%) to equity. The majority (80.64\%) used qualitative approach. More studies are needed in order to assess whether health systems had measurable impacts in terms of access and equity.

KEYWORDS Access to health services. Equity. Primary Health Care. 


\section{Introdução}

Acesso a serviços de saúde e equidade estão profundamente relacionados à capacidade de os sistemas de saúde se organizarem de modo a responder adequadamente às necessidades dos cidadãos. Starfield (2002) distingue acessibilidade de acesso. A primeira refere-se às características da oferta; já o segundo relaciona-se à forma pela qual as pessoas percebem a acessibilidade. Outros autores, como Travassos e Martins (2004), também apontam que a forma como as pessoas percebem a disponibilidade de serviços afeta a decisão de procurá-los, sendo que essa percepção é influenciada pela experiência passada com os serviços de saúde.

Goddard e Smith (2001) destacam o fato de que a disponibilidade de serviços pode também não ser do conhecimento de todos, e que diferentes grupos populacionais variam no grau de informação que possuem sobre os serviços a eles disponíveis. Desse modo, a experiência com os serviços e as informações que deles se dispõem influenciam a forma como as pessoas percebem as dificuldades/facilidades para obterem os serviços de saúde de que necessitam e, consequentemente, o acesso a eles (TRAVASSOS; MARTINS, 2004).

A efetiva implementação de redes de atenção à saúde no Sistema Único de Saúde (SUS), com centralidade na Atenção Básica, permite a ampliação do acesso e o uso regular de serviços de saúde com equidade. Considerando o direito universal à saúde, a redução das desigualdades deve ser política pública prioritária e pressupõe a garantia de acesso à rede ambulatorial e domiciliar com financiamento adequado, regulamentação e capacitação nos seus vários níveis de complexidade, ajustados às necessidades dos cidadãos, especialmente aqueles em maior situação de vulnerabilidade, tanto na rede pública quanto na rede privada (LOUVISON ET AL.2008).

Diferentes estudiosos, como Sousa e
Parreira (2010), Gil (2006) e Conill (2008), no Brasil, e, ainda, Tchouaket et al. (2012), no Canadá, têm destacado a importância de se fortalecer a Atenção Primária à Saúde (APS) para melhores resultados nos níveis de saúde da população e para que se tenha sistemas de saúde com melhor organização e desempenho. Entretanto, como bem destaca Starfield (2002), nem todos os países organizaram seus sistemas de saúde alicerçados fortemente na atenção primária, a despeito das recomendações da Organização Mundial de Saúde (OMS).

A OMS, em 1978, por ocasião da Conferência de Alma-Ata, expressou alguns valores que orientam a base conceitual dos cuidados primários de saúde afirmando: (1) que a saúde é um direito humano fundamental, e a consecução do mais alto nível possível de saúde é a mais importante meta social mundial, cuja realização requer a ação de outros setores sociais e econômicos, além do setor saúde; (2) que a chocante desigualdade existente no estado de saúde dos povos, particularmente entre os países desenvolvidos e em desenvolvimento, assim como dentro dos países, é política, social e economicamente inaceitável; (3) que esses cuidados primários fazem parte integrante tanto do sistema de saúde do país, do qual constituem a função central e o foco principal, quanto do desenvolvimento social e econômico global da comunidade. Representam o primeiro nível de contato dos indivíduos, da família e da comunidade com o sistema nacional de saúde, pelo qual os cuidados de saúde são levados o mais proximamente possível aos lugares onde pessoas vivem e trabalham, e constituem o primeiro elemento de um continuado processo de assistência à saúde (OMS, 1978).

Starfield (2002) enuncia as principais funções da APS, destacando a de constituir-se em porta de entrada do serviço, de modo a ser acessível à população, configurando-se como o primeiro recurso a ser buscado; a de assegurar a continuidade do cuidado e a de exercer a coordenação do cuidado, ainda que 
parte dele deva ser obtido em outros níveis de atendimento.

O presente trabalho tem por objetivo conhecer os estudos já realizados sobre o binômio acesso aos serviços de saúde e equidade, buscando artigos já publicados em países selecionados e que constituem, em sua maioria, a base para o trabalho intitulado 'Da política institucional aos processos do cuidar: estudos comparados sobre as práticas de promoção da saúde nas equipes do Programa Saúde da Família-PSF no Brasil e seus similares em Cuba, Canadá, Chile, Peru, Venezuela, Portugal e Colômbia'.

\section{Metodologia}

Trata-se de uma pesquisa de revisão estruturada da literatura científica com o objetivo de buscar os estudos realizados sobre acesso aos serviços de saúde e equidade, através de artigos publicados em diferentes países da Europa e das Américas. Alavi e Carlson (1992) registram que a revisão de literatura constitui-se em estudo teórico ilustrativo, uma vez que busca compreender as pesquisas que funcionam como guia prático.

Lembram Marconi e Lakatos (1999) que a finalidade da pesquisa bibliográfica é a de colocar o pesquisador em contato direto com tudo o que foi escrito, dito ou filmado sobre um determinado assunto. Portanto, ela não é uma mera repetição do que já foi dito ou escrito sobre um determinado assunto, mas oferece a possibilidade de examinar um tema sob uma nova abordagem, o que pode trazer conclusões inovadoras.

$\mathrm{O}$ percurso metodológico consistiu em realizar uma revisão de literatura sobre acesso aos serviços de saúde e equidade em saúde, com artigos científicos publicados no período de 1993 a 2013, disponíveis na Biblioteca Virtual em Saúde (BVS) do Centro Latino-Americano e do Caribe de Informação em Ciências da Saúde (Bireme). A Biblioteca inclui várias bases de dados, como, por exemplo, a Literatura LatinoAmericana e do Caribe em Ciências da Saúde (Lilacs). Foram excluídos os artigos que não tiveram adequação ao tema, respeitando-se os critérios de inclusão, conforme apresentados a seguir.

Os critérios de inclusão foram estabelecidos com base em dois descritores que já existem na BVS: acesso aos serviços de saúde e equidade. Todas as publicações selecionadas são artigos científicos nos idiomas inglês, português ou espanhol que tenham como assunto principal 'Acesso aos serviços de saúde' e 'Equidade em saúde'.

$\mathrm{Na}$ estratégia de busca, o tema foi delimitado por meio dos critérios de inclusão, respeitando-os rigorosamente e inserindo como primeiro filtro os artigos disponíveis por completo. No país/região como assunto, foram selecionados os seguintes países: Brasil, Canadá, Cuba, Chile, Colômbia, Peru, Portugal, Venezuela e Espanha. Seguiu-se a ordem da filtragem: texto completo (disponível); assunto principal (acesso aos serviços de saúde e equidade em saúde); tipo de documento (artigo); idioma (português, inglês e espanhol); país/região como assunto (Brasil, Canadá, Cuba, Chile, Colômbia, Peru, Portugal, Venezuela e Espanha) e ano de publicação (1993 a 2013).

Quando colocados os descritores na BVS (acesso aos serviços de saúde; equidade), foram encontrados 320 estudos. Após o primeiro filtro (texto completo disponível), foram reduzidos a 192 estudos. Ao buscar os assuntos principais, o número reduziu-se a 127. Posteriormente, quando se inseriu o filtro 'país como assunto', tivemos 59 estudos. Os artigos encontravam-se nos idiomas inglês, português ou espanhol, conforme critérios já estabelecidos. Com o filtro que seleciona o período de publicações escolhido, entre os anos de 1993 e 2013, foram obtidos 53 estudos. Por último, ao selecionar-se o tipo de documento (artigos científicos), o número foi reduzido a 39. Destes, restaram 31 artigos, que se enquadravam em todos os 
critérios que haviam sido definidos anteriormente ao começo da pesquisa.

Os artigos foram lidos ao longo de três meses e agrupados numa tabela Excel, constando título, ano, fonte, base, autor, idioma, tema, metodologia e resumo, referentes aos artigos encontrados nas bases já citadas. Finalmente, buscou-se identificar a natureza e a origem dessa produção. $\mathrm{O}$ referido projeto de pesquisa foi devidamente aprovado pelo Comitê de Ética em Pesquisa da Faculdade de Ciências da Saúde (FS), sob número 084/2012.

\section{Resultados e discussões}

Quanto à natureza, foram encontrados 24 (77,4\%) estudos qualitativos; dois quantitativos; dois qualiquantitativos; uma pesquisa avaliativa; um estudo etnográfico; e um estudo de casos múltiplos holístico, de abordagem qualitativa, num total de 31 trabalhos. A quase totalidade encontra-se em português (29), e apenas um em inglês e um em espanhol. A distribuição dos artigos por periódico de publicação encontra-se no gráfico 1, num total de 16 diferentes revistas científicas.

A pesquisa qualitativa é traduzida por aquilo que não pode ser mensurável, pois a realidade e o sujeito são elementos indissociáveis. Assim sendo, quando se trata do sujeito, levam-se em consideração seus traços subjetivos e suas particularidades. Tais pormenores não podem ser traduzidos em números quantificáveis, com bem ensina Minayo (2006).

Gráfico 1. Distribuição dos artigos por periódico de publicação

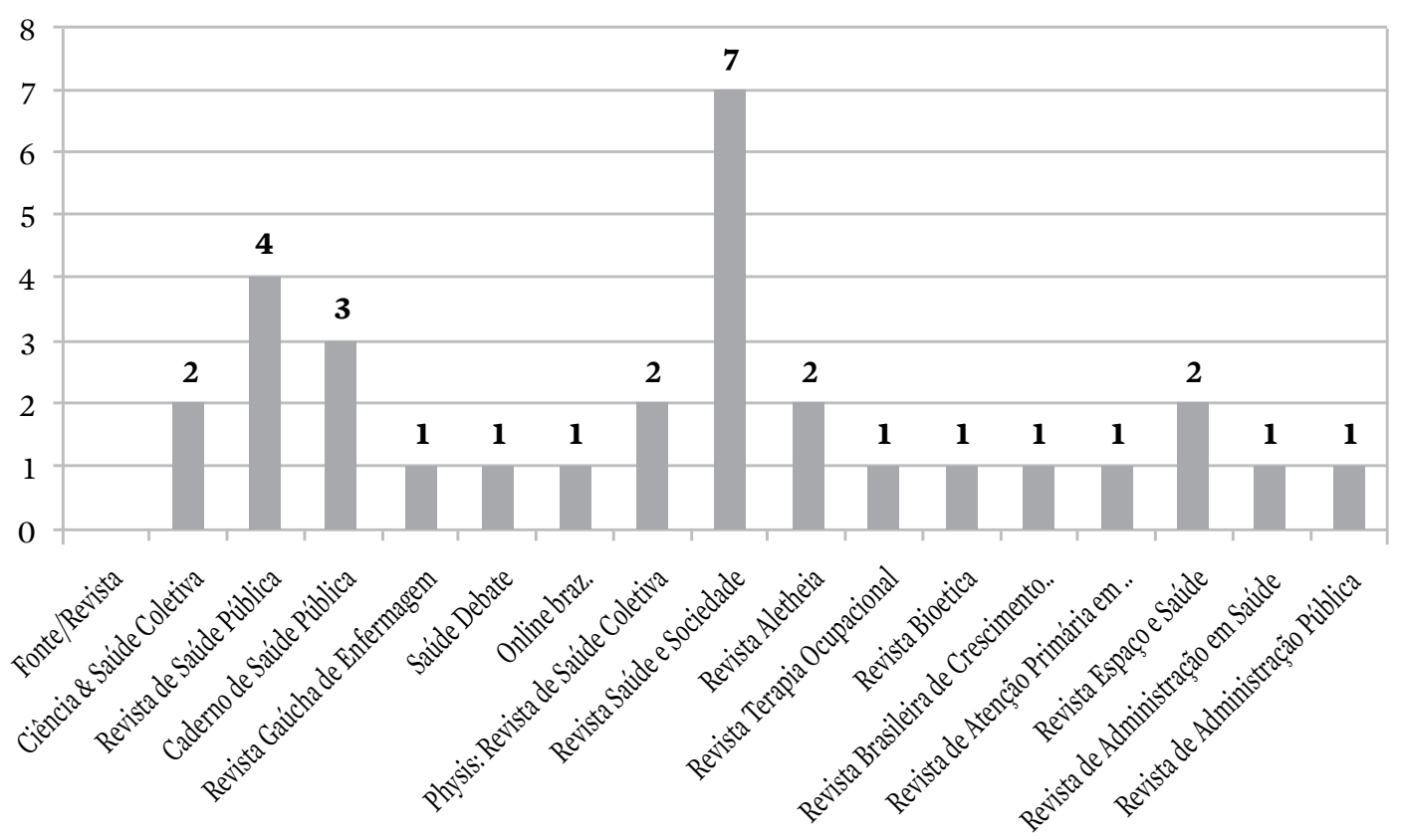

Fonte: Elaboração própria. 
Quanto ao eixo temático, encontrou-se um total de $18(58,06 \%)$ artigos nitidamente relacionados à organização de serviços de saúde e $13(41,94 \%)$ voltados ao tema da equidade. Não houve artigos que abordassem conjuntamente os temas 'acesso' e 'equidade', nem o tema 'acesso' isoladamente. Entre os primeiros, predominam os seguintes assuntos: saúde bucal; organização da atenção a pacientes crônicos; modo de funcionamento das equipes e de unidades de saúde da família. Já nos últimos estão presentes os temas relativos a raça, gênero e idosos, sobretudo quanto às práticas de saúde com essas populações específicas.

Percebe-se que predominam abordagens pontuais sobre experiências isoladas, distribuídas em diferentes temas que enfocam as questões de acesso e equidade numa perspectiva local, seja em modos de organização de serviços, seja com relação a práticas de saúde direcionadas a populações individualizadas ou vulneráveis.

A distribuição dos artigos por ano de publicação encontra-se no gráfico 2 e demonstrou que os anos de 2007, 2010 e 2011 foram os mais pródigos na produção de artigos sobre os temas em estudo.

Gráfico 2. Distribuição dos artigos por ano de publicação ição dos artigos por periódico de publicação

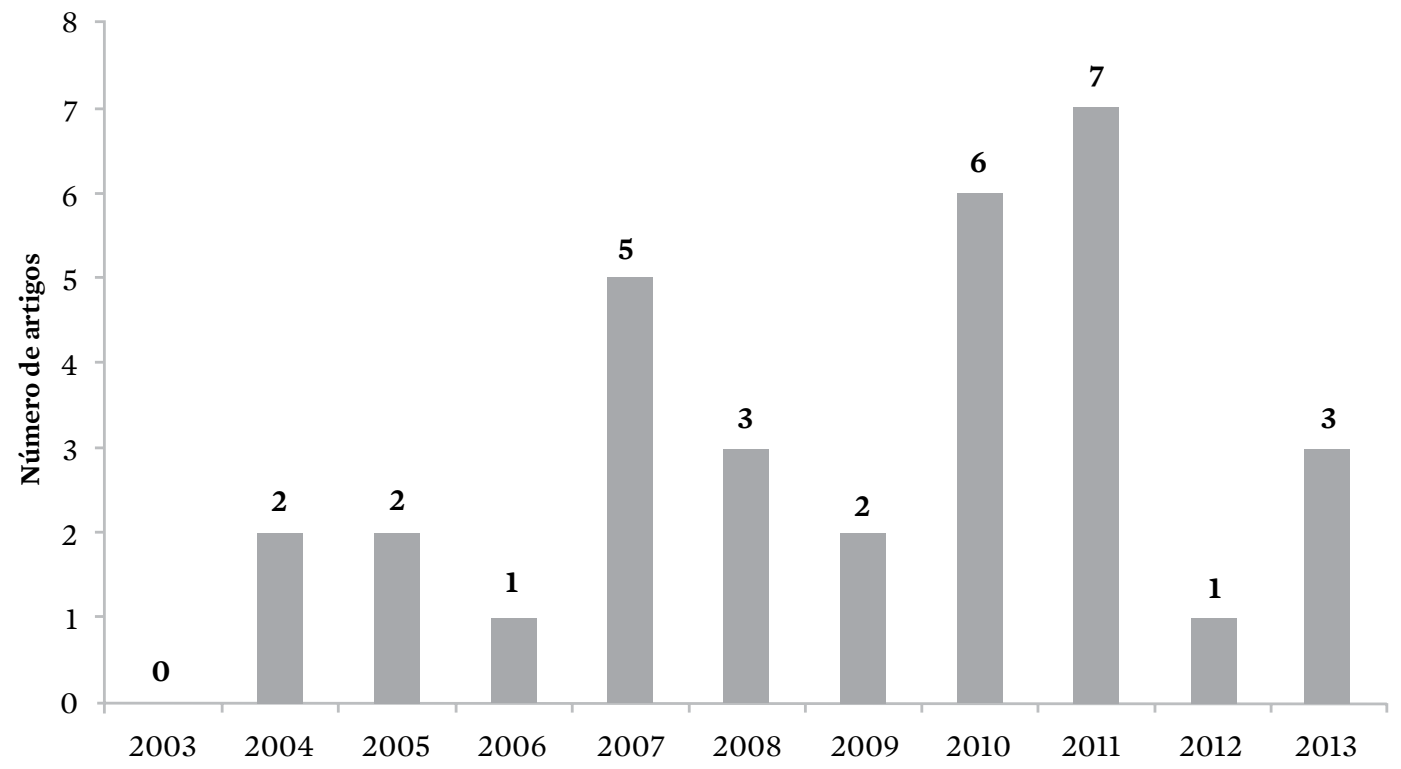

Fonte: Elaboração própria.

Percebe-se que no universo dos artigos analisados, há uma manifesta preocupação com aspectos relativos à equidade (41,94\% do total de publicações analisadas). Há de se observar que a equidade, ainda que citada com frequência entre os princípios ou diretrizes do SUS, a rigor, não tem, pelo menos de forma explícita, a mesma estatura jurídica dos demais (universalidade, integralidade, descentralização, participação social), como destacam Piola et al. (2009). Apesar disso, cada vez mais a preocupação 
com a equidade encontra-se presente no pensamento sanitário brasileiro.

Nos relatórios das Conferências Nacionais de Saúde de 1992, 1996 e 2000, por exemplo, a utilização do termo 'equidade', ou equivalente, é crescente. No relatório da IX Conferência (1992), aparece quatro vezes; no da X Conferência (1996), 14 vezes; e na XI Conferência (2000), 32 vezes (PINHEIRO; WESTPHAL; AKERMAN, 2009). Já no documento orientador para os debates da XIV Conferência, a preocupação com a equidade é o primeiro dos cinco tópicos propostos e intitula-se 'Avanços e desafios para a garantia do acesso e do acolhimento com qualidade e equidade' (CONFERÊNCIA NACIONAL DE SAÚDE, 2011).

No Brasil, há uma multiplicidade de entendimentos acerca do que é equidade em saúde, como atestam os trabalhos de Paim (2006); Granja, Zoboli e Fracolli (2013); Luiz (2005); Lucchese (2003) e Travassos e Castro (2012). Lembra Escorel (2001) que, em geral, o princípio da equidade tem sido operacionalizado em duas principais dimensões: condições de saúde e acesso e utilização dos serviços de saúde. No âmbito das condições de saúde, é analisada a distribuição dos riscos de adoecer e morrer em grupos populacionais. Embora variações biológicas, como sexo e idade, determinem diferenças de morbidade e mortalidade, a maior parte das condições de saúde é socialmente determinada e não decorre de variações naturais ou de livres escolhas pessoais por estilos de vida mais ou menos saudáveis. Os pobres, grupo social e economicamente vulnerável, pagam o maior tributo em termos de saúde, acumulando a carga de maior frequência de distribuição de doenças, sejam elas de origem infecciosa, crônico-degenerativas ou, ainda, as originadas de causas externas.

\section{Conclusão}

A produção científica encontrada nesta revisão, no que se refere aos trabalhos brasileiros, atesta que predominam temas locais que se, por um lado, podem ter validade como experiências exitosas, por outro, não se traduzem como prática homogênea no conjunto do sistema de saúde. Além disso, não enfocam os temas acesso e equidade conjuntamente, numa perspectiva sistêmica. Assim, importa aprofundar os estudos sobre acesso a serviços de saúde correlacionando-o à dimensão da equidade, pois os dois aspectos possuem íntima relação que importa decisivamente para a garantia da oferta de ações e serviços de saúde adequados, oportunos e capazes de responder às necessidades e expectativas dos cidadãos. É preciso, ainda, insistir sobre a necessidade de se analisar as dimensões conexas do acesso e da equidade numa perspectiva global, ou seja, de como tem sido ou não possível para o SUS, no seu todo, avançar nesses dois aspectos e produzir impactos mensuráveis. 


\section{Referências}

ALAVI, M.; CARLSON, P. A review of MIS research and disciplinary development. Journal of Management Information Systems, [s.l.] p. 45-62, 1992.

CONFERÊNCIA NACIONAL DE SAÚDE, 14., nov./ dez. 2011, Brasília. Documento orientador para os debates. Brasília, DF, 2011. Disponível em: <http:// conselho.saude.gov.br/14cns/doc_orientador.html>. Acesso em: 12 jun. 2014.

CONILL, E. M. Ensaio histórico-conceitual sobre a Atenção Primária à Saúde: desafios para a organização de serviços básicos e da Estratégia Saúde da Família em centros urbanos no Brasil. Cad. Saúde Pública, Rio de Janeiro, v. 24, supl.1, p. s7-s16, 2008.

ESCOREL, S. Os dilemas da equidade em saúde: aspectos conceituais. Brasília, DF: OPAS, 2001. Disponível em: $<$ http://biblioteca.planejamento.gov.br/bibliotecatematica-1/textos/saude-epidemias-xcampanhasdados-descobertas/texto-83-2013-os-dilemas-daequidade-em-saude-aspectos-conceituais.pdf $>$. Acesso em: 4 jun. 2014.

GIL, C. R. R. Atenção primária, atenção básica e saúde da família: sinergias e singularidades do contexto brasileiro. Cad. Saúde Pública, Rio de Janeiro, v. 22, n. 6 , p. 1171-1181, 2006

GODDARD, M.; SMITH, P. Equity of access to health care services: theory and evidence from the UK. Social Science \& Medicine, Oxford, v. 53, n. 9, p. 1149-1162, nov. 2001.

GRANJA, G. F.; ZOBOLI, E. L. C. P.; FRACOLLI, L. A. $O$ discurso dos gestores sobre a equidade: um desafio para o SUS. Ciência \&t Saúde Coletiva, Rio de Janeiro, v. 18, n. 12, p. 3759-3764, 2013.

LAKATOS, E. M.; MARCONI, M. A. Técnicas de pesquisa. 4. ed. São Paulo: Atlas, 1999.

LOUVISON, M. C. P. et al. Desigualdades no uso e acesso aos serviços de saúde entre idosos do município de São Paulo. Rev. Saúde Pública, v. 42, n. 4, p. 733-740,
2008. Disponível em: <http://www.scielo.br/pdf/rsp/ v42n4/6846.pdf >. Acesso em: 11 jul. 2016.

LUCCHESE, P. T. R. Equidade na gestão descentralizada do SUS: desafios para a redução de desigualdades em saúde. Ciência \&t Saúde Coletiva, Rio de Janeiro, v. 8, n. 2, p. 439-448, 2003.

LUIZ, O. C. Direitos e equidade: princípios éticos para a saúde. Arq. méd. ABC., Santo André, v. 30, n. 2, p. 69-75, 2005.

MARCONI, M. A.; LAKATOS, E. M. Técnicas de pesquisa. 3. ed. São Paulo: Atlas, 1999.

MINAYO, M. C. O Desafio do Conhecimento: Pesquisa Qualitativa em saúde. 9 ed. São Paulo: Hucitec, 2006.

ORGANIZAÇÃO MUNDIAL DE SAÚDE (OMS). Carta da Conferência de Alma-Ata, 1978. Disponível em: $<$ http://www.opas.org.br/declaracao-de-alma-ata/> . Acesso em: 23 maio 2015.

PAIM, J. S. Equidade e Reforma em Sistemas de Serviços de Saúde: o caso do SUS. Saúde e Soc., São Paulo, v. 15, n. 2, p. 34-46, 2006.

PIOLA, S. F. et al. Saúde no Brasil: algumas questões sobre o Sistema Único de Saúde (SUS). 2009. (Textos para Discussão, 139). Disponível em: <http://www.ipea. gov.br/portal/images/stories/PDFs/TDs/td_1391.pdf>. Acesso em: 12 jul. 2016.

PINHEIRO, M. C.; WESTPHAL, M. F.; AKERMAN, M. Equidade em saúde nos relatórios das conferências nacionais de saúde pós-Constituição Federal brasileira de 1988. Cad. de Saúde Pública, Rio de Janeiro, v. 21, n. 2, p. 449-458, 2005

SOUSA, M. F. Programa de Saúde da Família no Brasil - análise da desigualdade no acesso à atenção básica. Brasília, DF: Departamento de Ciência da Informação e Documentação da Universidade de Brasília, 2007. $250 \mathrm{p}$. 
SOUSA, M. F.; PARREIRA, C. M. F. Ambientes verdes e saudáveis: formação dos agentes comunitários de saúde na Cidade de São Paulo, Brasil. Rev. Panam. Salud Públ., Washington, DC, v. 28, n. 5, p. 399-404, 2010.

SOUSA, M. F. et al. Gestão da Atenção básica: redefinindo contexto e possibilidade. Divulgação em Saúde para Debate, Rio de Janeiro, n. 21, p. 7-14, 2000.

STARFIELD, B. Atenção primária: equilíbrio entre necessidades de saúde, serviços e tecnologia. Brasília, DF: Unesco; Ministério da Saúde, 2002. Disponível em: $<$ http://unesdoc.unesco.org/ulis/cgibin/ulis.pl?catno= 130805\&set=4BBCA640_1_386\&gp=1\&mode=e\&lin=1\& ll=1>. Acesso em: 14 jun. 2014.

TCHOUAKET, E. et al. Health care systems performance of $27 \mathrm{OCDE}$ contries. Int $J$ Health

PlannMgmt, Chichester, 2012.
TRAVASSOS, C.; CASTRO; M. S. M. Determinantes e desigualdades sociais no acesso e utilização dos serviços de saúde. In: GIOVANELLA, L. et al. (Org.). Políticas e Sistema de Saúde no Brasil. Rio de Janeiro: Fiocruz, 2012. p. 183-206.

TRAVASSOS, C.; MARTINS, M. Uma revisão sobre os conceitos de acesso e utilização de serviços de saúde. Cad. Saúde Pública, Rio de Janeiro, v. 20, Supl. 2, p. S190-S198, 2004.

Recebido para publicação em janeiro de 2016

Versão final em julho de 2016

Conflito de interesses: inexistente

Suporte financeiro: não houve 